\title{
Static Charge Fluctuations in Amorphous Silicon
}

\author{
L. Ley, J. Reichardt, and R. L. Johnson \\ Max-Planck-Institut für Festkörperforschung, D-7000 Stuttgart 80, Federal Republic of Germany
}

(Received 17 May 1982; revised manuscript received 27 September 1982)

\begin{abstract}
High-resolution Si $2 p$ core-level spectra reveal a homogeneous broadening for amorphous compared to crystalline silicon. This is due to static fluctuations in valence charges brought about by bond-length and bond-angle variations in the amorphous network. The observed Gaussian broadening of $256 \mathrm{meV}$ corresponds to mean deviation from charge neutrality of approximately 0.11 elementary charges. The addition of hydrogen reduces the charge fluctuations by $\sim 25 \%$.

PACS numbers: $72.80 . \mathrm{Ng}, 73.60 . \mathrm{Fw}, 79.60 . \mathrm{Eq}$
\end{abstract}

Amorphous solids exhibit a number of properties not usually found in their crystalline counterparts. For example, the electrons at the band edges of semiconductors are localized and contribute to the electric current via hopping between localized states-a process that dominates the transport of amorphous silicon or germanium at low temperatures. ${ }^{1}$

Another example of these unique properties is the excess specific heat and the reduced thermal conductivity of glasses such as $\mathrm{SiO}_{2}$ or $\mathrm{As}_{2} \mathrm{~S}_{3}$ at temperatures below $\sim 1 \mathrm{~K}$, that are most likely due to low-lying excitations connected with the rearrangement of structural units in the amorphous solids. ${ }^{2}$ Both phenomena are intimately connected with the loss of long-range order that can result in a certain flexibility of the amorphous network for glasses such as $\mathrm{SiO}_{2}$ or $\mathrm{As}_{2} \mathrm{~S}_{3}$.

The chemical nature of the bonding, i.e., covalent or ionic, however, was thought to be the same in crystalline and amorphous modifications of a material. This view relies on the experimental fact that the structural units- $\mathrm{Si}_{-}-\mathrm{Si}_{4}, \mathrm{Si}-$ $\mathrm{O}_{4}$ in the case of silicon or $\mathrm{SiO}_{2}$, for example -are maintained and that nearest-neighbor distances are found almost unchanged. In particular it was taken for granted that the homopolar nature of the $\mathrm{Si}-\mathrm{Si}$ bond is preserved in amorphous silicon. Guttman, Ching, and Rath, in their recent model calculations of the electron distribution in amorphous silicon $(a-\mathrm{Si})$, however, found net static charges on the atoms of an amorphous silicon cluster as a result of bond-length variations involving neighbors two or more atoms removed. The charges showed a distribution with standard deviation $\Delta q_{\mathrm{rms}}=0.2$ electrons. $^{3}$ Such charge fluctuations - if they exist - would explain the observed ir activity of the lattice modes in $a-\mathrm{Si}_{0}{ }^{4,5}$ They would also set up considerable electric fields within the bulk $a-\mathrm{Si}$ with consequences for the optical absorption and the charge trans- port. The Coulomb energy between these charges would furthermore contribute to the total energy of the amorphous network.

We report here direct experimental confirmation of these charge fluctuations and give numbers for the average deviation $\Delta q$ from neutrality in sputtered amorphous films of silicon. We further present evidence that these charges are reduced upon hydrogenation of $a-\mathrm{Si}$ in such a way that the reduction is large for atoms bonded directly to hydrogen and small but finite for all others.

The charges are determined through the chemical shift they induce in the binding energy of the Si $2 p$ core levels as measured in photoemission. The shift $\Delta E$ is approximately $2.2 \mathrm{eV}$ per unit charge removed or added to the valence shell of $\mathrm{Si}^{6}{ }^{6}$ The resolution necessary to detect shifts corresponding to $\Delta q$ of the order of $0.1 e$ was obtained with use of synchrotron radiation from the DORIS storage ring, Hamburg, in conjunction with a high-resolution grating monochromator. The Si $2 p$ doublet $[\Delta($ spin orbit $)=0.60 \mathrm{eV}]$ of a crystalline $\mathrm{Si}(111)$ surface excited with $h \nu=107.5$ $\mathrm{eV}$ is shown in Fig. 1(a) and is well described by the convolution of a Lorentzian $\left\{1 /\left[\left(\omega-\omega_{0}\right)^{2}+\Gamma^{2}\right]\right\}$ that takes into account the lifetime of the core hole $[2 \Gamma=210 \pm 30 \mathrm{meV}]$ and a Gaussian $\{\exp [-(\omega$ $\left.\left.\left.-\omega_{0}\right)^{2} / 2 \sigma^{2}\right]\right\}$ representing the combined resolution of monochromator and electron analyzer $\left(\sigma_{\exp }=60\right.$ $\mathrm{meV})$.

The corresponding spectrum [Fig. 1(b)] taken with $h \nu=110 \mathrm{eV}$ from an amorphous silicon sample prepared in situ by sputter deposition is considerably broader so that the spin-orbit components are no longer resolved. The data points are again fitted by the convolution of a Lorentzian and a Gaussian with parameters displayed in Table I. The width of the Gaussian is partitioned into a contribution $\sigma_{\exp }$ due to the experimental resolution obtained from a spectrum of crystalline 


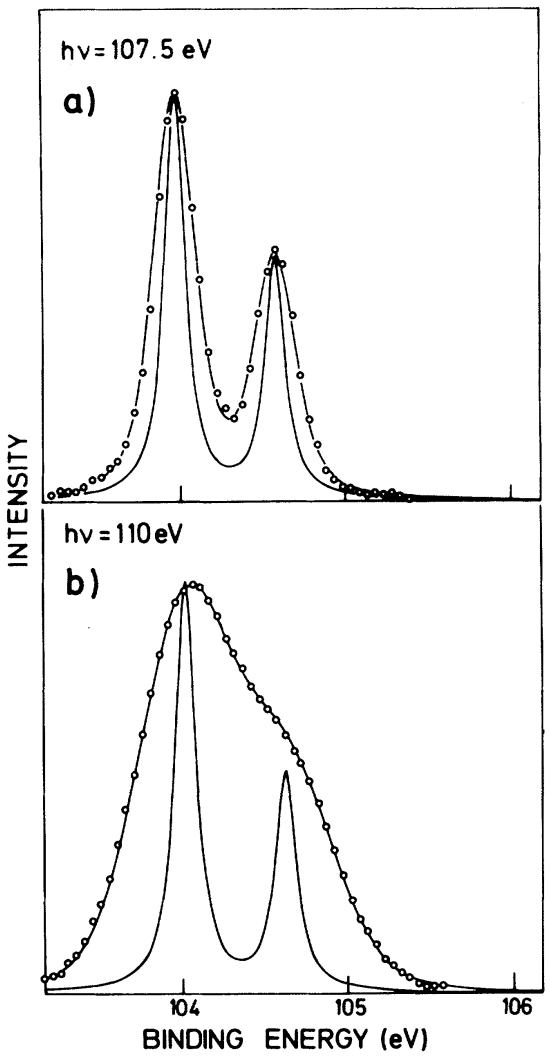

FIG. 1. Si $2 p$ core-level spectra of (a) crystalline and (b) amorphous silicon. Data points (circles), the result of a least-squares fit, and the Lorentzian contributions to the component lines are shown.

silicon $(c-\mathrm{Si})$ measured under identical conditions and a contribution $\sigma_{\text {amorph }}$ that takes the homogenous broadening into account such that $\sigma_{\exp }{ }^{2}$ $+\sigma_{\text {amorph }}{ }^{2}=\sigma^{2}$. The decrease in $2 \Gamma$ reflects a reduction in the Auger decay rate of the core holes most likely due to the changed electronic structure of the valence bands.

Band bending due to surface charges can be ruled out as the cause for the observed broadening. The binding energy of the core levels changed by less than $50 \mathrm{meV}$ for photon energies between 107.5 and $140 \mathrm{eV}$ corresponding to sampling depths between 30 and $8 \AA$, respectively. Similarly, unresolved chemical shifts due to contamination are not responsible. Aside from the fact that they would yield an asymmetric broadening towards higher binding energies, wide energy scans and valence-band spectra proved the sample of Fig. 1(b) to be free of any contamination including hydrogen.

We therefore conclude that the broadening is due to a distribution of chemically shifted $\mathrm{Si} 2 p$ lines, the shifts being due to a distribution of charges $\Delta q$ on the $\mathrm{Si}$ atoms as a result of the peculiarities of the $a$-Si structure. Two reasons for such charges come to mind. (i) There may be fluctuations in the bond lengths of the $a-\mathrm{Si}$ network as suggested by Guttman, Ching, and Rath. ${ }^{3}$ The dominant factor would then be the fluctuations in next-nearest-neighbor distance reflected in the nonnegligible variation in bond angle $\theta(\Delta \theta$ $\sim 10^{\circ}$ ). Nearest-neighbor distances are quite rigid and the overlap between wave functions of silicon atoms removed by more than one intermediate atom is too small to induce any significant charge transfer. If this point of view is correct, the charges should be distributed homogeneously throughout the amorphous specimen:

(ii) There is, on the other hand, ample evidence that $a-\mathrm{Si}$ is not homogeneous but contains a large volume fraction of voids enclosed by inner surfaces. ${ }^{7}$ It is therefore conceivable that

TABLE I. Parameters obtained in a least-squares fit of Si $2 p$ core levels. Errors in the last digits are given in parentheses.

\begin{tabular}{|c|c|c|c|c|c|c|}
\hline Sample & $\begin{array}{c}2 \Gamma \\
(\mathrm{meV})\end{array}$ & $\begin{array}{c}\sigma_{\mathrm{am}} \\
(\mathrm{meV})\end{array}$ & $\begin{array}{c}\Delta q_{\mathrm{rms}} \\
|e|\end{array}$ & $\begin{array}{c}\Delta \boldsymbol{E}_{\mathrm{H}} \\
(\mathrm{meV})\end{array}$ & $\begin{array}{c}\Delta q(\mathrm{H}) \\
|e|\end{array}$ & $\begin{array}{c}C_{\mathrm{H}} \\
\text { at.\% }\end{array}$ \\
\hline$a-\mathrm{Si}$ & $106(40)$ & $256(10)$ & 0.11 & $\cdots$ & $\cdots$ & $\cdots$ \\
\hline $\begin{array}{l}a-\mathrm{Si}: \mathrm{H} \text {, four doublets } \\
\text { (a) Equal width }\end{array}$ & $106(40)$ & $186(10)$ & 0.08 & $330(10)$ & 0.15 & 56; $\begin{aligned} & 21 \mathrm{Si}-\mathrm{H} \\
& 18 \mathrm{Si}-\mathrm{H}_{2} \\
& 17 \mathrm{Si}-\mathrm{H}_{3}\end{aligned}$ \\
\hline \begin{tabular}{l|l}
$\mathrm{Si}$ & $\mid$ \\
$\mathrm{Si}-\mathrm{H}$ & \\
$\mathrm{Si}-\mathrm{H}_{2}$ & $\mathbf{S}$ \\
$\mathrm{Si}-\mathrm{H}_{3}$ &
\end{tabular} & $106(40)$ & $\begin{array}{r}192(10) \\
128 \\
64 \\
0\end{array}$ & $\begin{array}{c}0.09 \\
0.06 \\
0.03 \\
0\end{array}$ & $340(10)$ & 0.15 & $\begin{array}{ll}48 ; & 16.6 \mathrm{Si}-\mathrm{H} \\
19.5 \mathrm{Si}-\mathrm{H}_{2} \\
12 \quad \mathrm{Si}-\mathrm{H}_{3}\end{array}$ \\
\hline
\end{tabular}


the broadening of the Si $2 p$ levels in $a-S i$ reflects a distribution of "surface" peaks similar to those obtained for $c$-Si surfaces. ${ }^{8}$ Because the surface atoms on the inner surfaces do not occupy welldefined and ordered positions as they do on the $\mathrm{Si}(111)$ face we might expect a range of surface shifts reflecting the disordered nature of the underlying "bulk." The magnitude of the surface shift found in $c$-Si would nicely account for the observed broadening in $a-\mathrm{Si}$.

A decision between the two models can be made based on the results of hydrogenated $a-\mathrm{Si}(a-\mathrm{Si}$ : $\mathrm{H})$. The incorporation of hydrogen during the deposition of $a-\mathrm{Si}$ is known to form $\mathrm{Si}-\mathrm{H}$ bonds and thereby to saturate dangling bonds at the inner void surfaces as well as other defects.

The Si $2 p$ core-level spectra $(h \nu=110 \mathrm{eV})$ of hydrogenated $a-\mathrm{Si}$ (prepared by adding $50 \mathrm{vol} \% \mathrm{H}_{2}$ to the sputter gas) are compared with that of unhydrogenated $a$-Si in Fig. 2(a). The leading (low binding energy) edge of the spectrum of $a-\mathrm{Si}: \mathrm{H}$ is clearly steeper than that of $a-\mathrm{Si}$ and extra intensity due to $\mathrm{Si}-\mathrm{H}_{x}$ configurations is found towards higher binding energy. From infrared absorption data and valence-band spectra of the same specimens we know that these films contain $\mathrm{Si}-\mathrm{H}, \mathrm{Si}-$ $\mathrm{H}_{2}$, and $\mathrm{Si}-\mathrm{H}_{3}$ units adding up to a total bulk hydrogen concentration of $(35 \pm 5)$ at. $\%$ We have therefore fitted the spectrum of Fig. 2(a) with a total of four doublets, assuming a constant shift $\Delta E_{\mathrm{H}}$ per attached hydrogen atom in accordance with the additivity of chemical shifts that worked well for $a$-Si containing the much more electronegative fluorine. ${ }^{9}$ The lifetime broadening $\Gamma$ of all lines was assumed the same as that of $a-\mathrm{Si}$. Two equally satisfactory fits were obtained (see Table I); one where the widths of all components were the same, and one where we assumed that the amorphous broadening decreases in proportion to the number of hydrogen atoms attached to a silicon atom. The latter fit is shown in Fig. 2(b). It implies that the constraints on the $\mathrm{Si}$ bonds are gradually relaxed as one, two, or three bonds are released from the network. We favor this fit because the hydrogen content obtained from the properly weighted contribution of the shifted components to the total spectrum is in closer agreement with the ir value than the 56 at.\% obtained for the other fit of Table I. The remaining discrepancy is due to a surface enrichment of hydrogen that will be discussed elsewhere.

The reduction in the amorphous broadening upon hydrogenation is determined by the leading

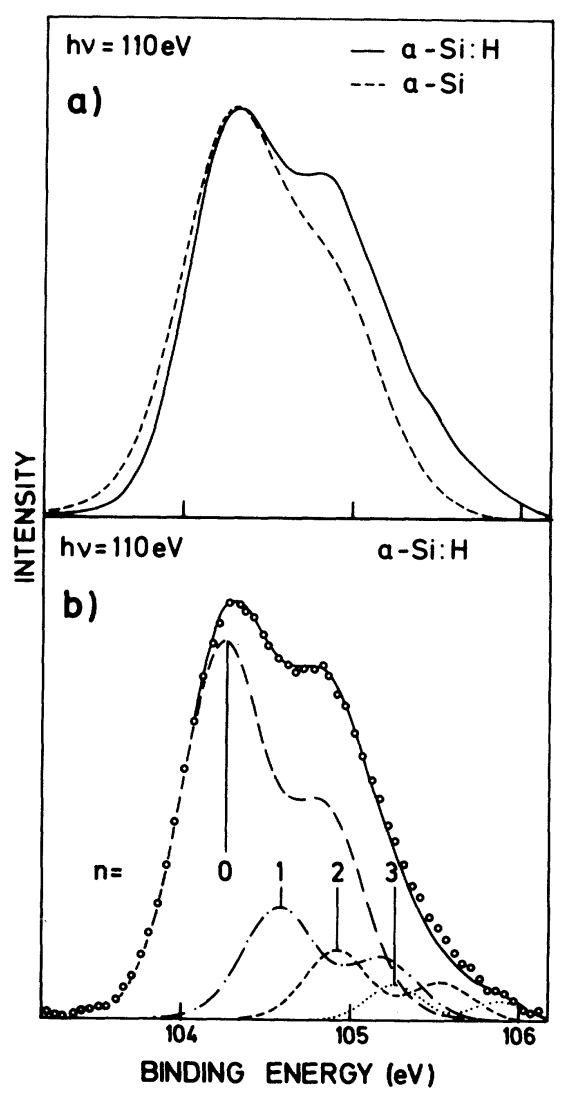

FIG. 2. (a) Comparison of the Si $2 p$ core-level spectra of unhydrogenated $(a-\mathrm{Si})$ and hydrogenated $(a-\mathrm{Si}: \mathrm{H})$ amorphous silicon. The latter is shifted by $0.2 \mathrm{eV}$ towards lower binding energy to facilitate comparison. The $0.2 \mathrm{eV}$ reflects a corresponding difference in the Fermi-level position. (b) Least-squares fit of the $a-\mathrm{Si}: \mathrm{H}$ spectrum of (a) to four doublets corresponding to $\mathrm{Si}$ atoms bonded to $n=0,1,2$, or 3 hydrogen atoms, respectively.

edge of the spectrum and is thus virtually the same for both fits as is the shift per attached hydrogen atom $\Delta E_{\mathrm{H}}=335 \pm 10 \mathrm{meV}$. It corresponds to a charge transfer of $\Delta q(\mathrm{H})=0.15$ electron from Si to hydrogen, noticeably larger than expected on the basis of Pauling's electronegativity $[\Delta q(\mathrm{H})$ $=0.02]$. Values of $\Delta q(\mathrm{H})$ comparable to ours were, however, obtained by Kelfve et al. ${ }^{10}$ from studies of gaseous $\mathrm{Si}$ compounds $[\Delta q(\mathrm{H})=0.12]$ and by Kramer, King, and McKinnon ${ }^{11}$ from model calculations on $a-\mathrm{Si}: \mathrm{H}$ clusters $[\Delta q(\mathrm{H})=0.27]$.

The main result of the investigation of $a-\mathrm{Si}: \mathrm{H}$ is that the broadening of the Si core levels is reduced in proportion to the number of hydrogen atoms attached to each $\mathrm{Si}$ atom. The width for silicon atoms not bonded directly to hydrogen is only slightly reduced. This excludes the assump- 
tion that all broadening in $a-\mathrm{Si}$ is due to surface chemical shifts of atoms surrounding voids. It must rather be attributed to random charge fluctuations as the result of bond length variations in the amorphous network. The average rms charge deviation is estimated to be 0.11 electron in $a-\mathrm{Si}$, only about half as much as obtained by Guttman, Ching, and Rath. In $a-\mathrm{Si}: \mathrm{H}$ the incorporation of hydrogen leads apparently to an overall reduction in the bond length fluctuations as manifested in the reduced $\sigma_{\text {amorph }}$, corresponding to charge fluctuations of only 0.09 electron. This is in agreement with the $20 \%$ attenuation of the TO bands in the ir spectrum of $a-\mathrm{Si}$ upon hydrogenation. ${ }^{5}$

We thank the staff of Hasylab for their kind hospitality and in particular C. Kunz for making his spectrometer available to us.

\footnotetext{
${ }^{1}$ See, e.g., N. F. Mott and E. A. Davis, Electronic Processes in Non-crystalline Materials (Clarendon, Oxford, 1979).

${ }^{2}$ P. W. Anderson, B. I. Halperin, and C. M. Varma,
}

Philos. Mag. 25, 1 (1972).

${ }^{3}$ L. Guttman, W. Y. Ching, and J. Rath, Phys. Rev. Lett. $\underline{44}, 1513$ (1980).

${ }^{4}$ See, e.g., M. Brodsky and A. Lurio, Phys. Rev. B 9, 1646 (1974).

${ }^{5}$ S. C. Shen, C. J. Fang, M. Cardona, and L. Genzel, Phys. Rev. B 22, 2913 (1980).

${ }^{6}$ F. J. Grunthaner, P. J. Grunthaner, R. P. Vasquez, B. F. Lewis, J. Maserjian, and A. Madhukar, Phys. Rev. Lett. $\underline{43}, 1683$ (1979).

${ }^{7}$ S. C. Moss and J. F. Graczyk, Phys. Rev. Lett. 21, 1575 (1969).

${ }^{8}$ There is as yet no general agreement on the exact magnitude of the surface shift on $\mathrm{Si}(111)$. See, e. g., S. Brennan, J. Stöhr, R. Jaeger, and J. E. Rowe, Phys. Rev. Lett. 45, 1414 (1980), and F. J. Himpsel, P. Heimann, T. C. Chiang, and D. E. Eastman, Phys. Rev. Lett. 45, 1112 (1980).

${ }^{9}$ K. J. Gruntz, L. Ley, and R. L. Johnson, Phys. Rev. B 24, 2069 (1981).

${ }^{10} \mathrm{P}$. Kelfve, B. Blomster, H. Siegbahn, K. Siegbahn, E. Sanhueza, and O. Goscinski, Phys. Scr. 21, 75 (1980).

${ }^{11}$ B. Kramer, H. King, and A. McKinnon, in Proceedings of the Sixteenth International Conference on the Physics of Semiconductors, Montpellier, 1982 (to be published). We thank the authors for private communication of their results.

\title{
Thermal Excitation of Two-Dimensional Plasma Oscillations
}

\author{
Ralph A. Höpfel, Erich Vass, and Erich Gornik \\ Institut für Experimentalphysik, Universität Innsbruck, A-6020 Innsbruck, Austria
}

(Received 16 August 1982)

\begin{abstract}
Experimental evidence for thermal excitation of plasmons in two-dimensional electron systems is reported. The spectral intensity and temperature dependence of the thermal excitation are calculated by means of Bose-Einstein statistics, and give quantitative agreement with the results of far-infrared emission experiments. It is found that the plasmons may contribute significantly to the specfic heat of the two-dimensional electron system at low electron densities and high temperatures.
\end{abstract}

PACS numbers: $73.40 . \mathrm{Qv}, 65.40 .-\mathrm{f}, 71.45 . \mathrm{Gm}, 72.30 .+\mathrm{q}$

Longitudinal plasma waves in two-dimensional electron systems (2D plasmons) exhibit a different dispersion behavior from 3D carrier systems and have therefore been the subject of intense experimental and theoretical investigations. ${ }^{1-5}$ The dispersion relation $\omega(k)$ has been verified with high accuracy by far-infrared (FIR) absorption ${ }^{6}$ and emission, ${ }^{7}$ and recently by light-scattering ${ }^{8}$ experiments. For strictly two-dimensional plasmons one obtains a dispersion relation ${ }^{5} \omega$ proportional to $k^{1 / 2}$; for coupled plasmon modes of lay- ered $2 \mathrm{D}$ electron systems the frequency is approximately a linear function ${ }^{8}$ of the wave vector $k$. In the present work it will be shown that plasmons in 2D and layered electron systems can be excited thermally by heating the electron gas with an external electric field. The spectral intensity of the excitation will depend on the temperature and density of the plasma.

The spectral intensity of the longitudinal twodimensional plasmons is calculated with BoseEinstein statistics. ${ }^{9}$ The criterion for well-de- 